\title{
Features of Microwave Magnetic Dynamics in Nanostructures with Strong Spin-Orbit Interaction
}

\author{
A. M. Korostil and M. M. Krupa \\ Institute of Magnetism, N.A.S. and M.E.S. of Ukraine, \\ $36^{\underline{b}}$ Academician Vernadsky Blvd., \\ UA-03680 Kyiv-142, Ukraine
}

Features of the current and spin-orbit-coupling induced magnetic dynamics in multilayer nanostructures with nonmagnetic heavy-metal layers possessing by a strong spin-orbit coupling are studied. The spin Hall effect of the conversion of an incoming charge current into a transverse (with respect to the charge current) spin current impacting on the magnetic dynamics through a spin-transfer torque provides the excitation of the magnetic dynamics including magnetic precession and switching. The magnetodynamic effect of a spin-current pumping generation together with the inverse spin Hall effect of conversion of the spin current into the incoming charge current provide the influence of the magnetic dynamics on the incoming charge current. These feedforward and feedback between the incoming charge current and the magnetic dynamics can be the basis for the spin-orbit-driven self-sustained and auto-oscillations of a magnetic order in ferro- and antiferromagnetics layers of the nanostructures. As shown, the considered magnetic nanostructures can possess by properties of controlled microwave radiation attaining tens $\mathrm{THz}$ in the antiferromagnetic case.

Вивчаються особливості магнетної динаміки, індукованої вхідним зарядовим струмом і спін-орбітальною взаємодією у багатошарових наноструктурах з немагнетними прошарками на основі важких металів із сильною спін-орбітальною взаємодією. Спіновий Голлів ефект перетворення вхідного зарядового струму в поперечний (відносно зарядового струму) спіновий струм, діючий на магнетну динаміку через передавання спінового крутильного моменту, забезпечує збудження магнетної динаміки, включаючи прецесію та перемикання. Магнетодинамічний ефект ґенерування спінового струму помпування разом зі зворотнім Голловим ефектом перетворення спінового струму в зарядовий струм забезпечує вплив магнетної динаміки на вхідний зарядовий струм. Такі прямий і зворотній впливи між вхідним зарядовим струмом і магнетною динамікою можуть становити основу спін-орбітально керованої ос- 
циляції магнетного порядку в феро- або антиферомагнетних прошарках наноструктур. Показано, що досліджувані магнетні наноструктури можуть мати властивості контрольованого мікрохвильового випромінення, що може сягати десятків ТГц у випадку антиферомагнетних матеріялів.

Изучаются особенности магнитной динамики, индуцируемой входным зарядовым током и спин-орбитальным взаимодействием в многослойных наноструктурах с немагнитными прослойками на основе тяжёлых металлов с сильным спин-орбитальным взаимодействием. Спиновый эффект Холла превращения входного зарядового тока в поперечный (относительно зарядового тока) спиновый ток, действующий на магнитную динамику через передачу спинового крутильного момента, обеспечивает изменение магнитной динамики, включающей прецессию и переключение. Магнитодинамический эффект генерирования спинового тока накачки вместе с обратным эффектом Холла превращения спинового тока в зарядовый ток обеспечивает влияние магнитной динамики на входной зарядовый ток. Такие прямое и обратное воздействия между входным зарядовым током и магнитной динамикой могут представлять основу для спин-орбитально управляемой осцилляции магнитного порядка в ферро- или антиферромагнитных прослойках. Показано, что исследуемые магнитные наноструктуры могут обладать свойствами контролируемого микроволнового излучения, достигающего десятков ТГц в случае антиферромагнитных материалов.

Keywords: magnetic nanostructures, spin Hall effect, spin transfer torque, magnetic dynamics, spin pumping effect, spin current, inverse spin Hall effect.

Ключові слова: магнетні наноструктури, спіновий Голлів ефект, спіновий крутильний момент, магнетна динаміка, ефект спінового помпування, спіновий струм, обернений спіновий Голлів ефект.

Ключевые слова: магнитные наноструктуры, спиновый эффект Холла, спиновый крутильный момент, магнитная динамика, эффект спиновой накачки, спиновый ток, обратный спиновый эффект Холла.

(Received June 30, 2016)

\section{INTRODUCTION}

There is much current interest in dynamical processes in magnetically ordered systems both from scientific and technological viewpoints. The special interest is related to the problem of the intercoupling between a spin-polarized electron current and the magnetic dynamics in multilayer magnetic nanostructures that can be exhibited in such phenomena, as magnetic switching and a sustained precession of magnetic-order vectors. 
The interrelation between the spin-polarized current and magnetic order vectors in magnetic multilayer nanostructures, permitting their mutual control, constitutes the basis of the operation of novel nanodevices with properties of a magnetic random-access memory (MRAM), magnetic logic and coherent microwave radiation sources that presents considerable fundamental and application interest [1-8]. The operation of these devices relies on the spinpolarized current-induced magnetization switching together with tunnel magnetoresistance effect and the like induced magnetization precession. Such phenomena have real potential for application in systems of high-speed magnetic processing information and high frequency fine-tuned $\mathrm{GHz}$ and $\mathrm{THz}$ electromagnetic radiation.

The intercoupling between a spin current and magnetic state in magnetic nanostructures constitutes the basis of the currentinduced manipulation by magnetic dynamics and vice-versa, i.e. the magnetic state-induced manipulation by the spin current [9, 10]. The spin current can be converted from an incoming charge current under internal effective magnetic fields of interactions of a different origin (including $s-d$ exchange and spin-orbit interactions) with corresponding features of the action of a spin torque on the magnetic states and their dynamics. Inducing magnetic dynamics, such a spin torque can cause switching and precession of the magnetic order vectors (including ferro- and antiferromagnetic orders) in magnetic nanolayers with ferromagnetic (FM) and antiferromagnetic (AF) interactions. The frequency of the magnetic dynamics is determined by the magnitude of magnetic exchange interaction, which is the largest for antiferromagnetic materials. The prospect of obtaining the technological magnetic nanostructures with low threshold incoming currents, low power consumption and controlled high frequency operation is related to utilization of the spin-orbit effects of the spin polarization and magnetic nanostructures with AF exchange interactions.

Generally, the spin-orbit interaction includes the bulk spin Hall (SH) effect $[7,8]$ of the transverse (relatively to an incoming current) deflection of electrons with opposites spins in opposite sides and the interface (two-dimensional) Rashba spin-orbit (RSO) effect $[9,10]$ of the spin splitting of an electron disperse along an electron wave vector. The impact of the spin current on the magnetic states realizes via the spin torque $T$ consisting of so-called a field-like and dumping-like part $T_{\|}$and $T_{\perp}$, respectively, which are related to the effects of magnetic-order switching and precession dumping or antidumping. The field-like torque $T_{\|}$originates predominantly by the spin-orbit coupling at the interface in combination with the perturbation of the electron distribution function. The torque $T_{\perp}$ originates predominantly by the perturbation of electronic states by the 
applied electric field.

The current spin-orbit-controlled microwave magnetic dynamics is realized for nanostructures composed of a heavy metal nanolayer (for instance, $\mathrm{Pt}, \mathrm{Ta}$ ) possessing by the strong enough spin-orbit interaction and the adjacent active magnetic nanolayer with a strong exchange interaction attaining maximum values of the order of tens $\mathrm{THz}$ in the $\mathrm{AF}$ cases. For multisublattice magnetic structures (for instance, for AFs), a general magnetic dynamics is a combined effect of dynamics of each of magnetic sublattices coupled by the strong exchange interaction. The interconnection between the incoming charge current and magnetic dynamics occurs in the mentioned case via the spin current and the spin transfer effect for the each sublattice singly. The mentioned magnetic systems with feedback, realizing via the direct and inverse $\mathrm{SH}$ effects of the spin pumping with spin backflow, possess by properties of sustained steady-state microwave spin torque oscillations, convertible via a magnetoresistance effect into an ac voltage and high-frequency radiation.

This paper is organized as follows. In section 2, the conversion of an incoming current into the spin current under internal effective bias fields of the exchange interactions of a different origin in magnetic nanostructures is studied. In Section 3, the spin and charge density diffusions in the bilayer magnetic structures with the strong spin-orbit interaction are considered in the framework of nonlinear kinetic leading to renormalization of parameters of a magnetic precession. Section 4 is devoted to dynamic feedback in $\mathrm{F} / \mathrm{SH}$ nanostructures. It is shown that spin pumping and spin transfer torques as two reciprocal processes result in a dynamical feedback effect interconnecting energy dissipation channels of both magnetization and current. In Section 5, features of spin pumping and spin-transfer torques as two reciprocal phenomena are considered in AF-based nanostructures. In Section 6, the current-induced magnetic dynamics is considered in the bilayer nanostructures composed of an insulating $\mathrm{AF}$ and adjusted heavy normal metal with the spin Hall effect. It is shown that the combined effect of current-induced torque and spin pumping introduces a dynamical feedback that sustains steady-state oscillations with amplitudes controllable via the applied current.

\section{THE SPIN POLARIZATION AND SPIN CURRENT}

The electric control of the magnetic dynamics in the mentioned magnetic nanostructures occurs through the exchange interaction between the spin current and a localized magnetic order. The spin current induces the spin torque causing magnetic dynamics in the 
form of the precession or switching of the magnetization in Fs and AF order in AFs [11-13]. The magnitude of the spin torque is determined by the mechanism of the conversion of incoming charge current into the spin current interacting with the magnetic order via an exchange interaction. The conversion of the incoming electric current into spin polarization state in the ferromagnetic nanostructures can be produced by the effective bias field of the $s-d$ exchange interaction in the magnetic layer acting as a spin polarizer. The spin polarization occurs as the results of the spin splitting of the electron band spectrum on the two branches with and without their intersection by the Fermi level (Fig. 1) (see [6]) that correspond two (Fig. 1,b) and single (Fig. 1, c) channel conductions of the electrons with different spin projections relatively to the magnetization.

In the usual two-channel case, the majority electros with the spin projection parallel to the magnetization, occupy the one conduction channel and the minority electrons with the antiparallel spin projection occupy the another conduction channel. This results in the incomplete spin polarization of the electric current. In the singlechannel case, which is realized for magnetic semi-metals [6], the conduction electrons with the fixed spin projection occupy only one conduction band and the incoming current converts into the pure spin current.

The impact of the polarized electric current on the magnetization occurs through the exchange interaction between the corresponding spin polarized current and the controlled localized spins. The passage of the spin-polarized current into the controlled magnetic nanolayer causes the spin torque exerting the magnetization switching or precession.
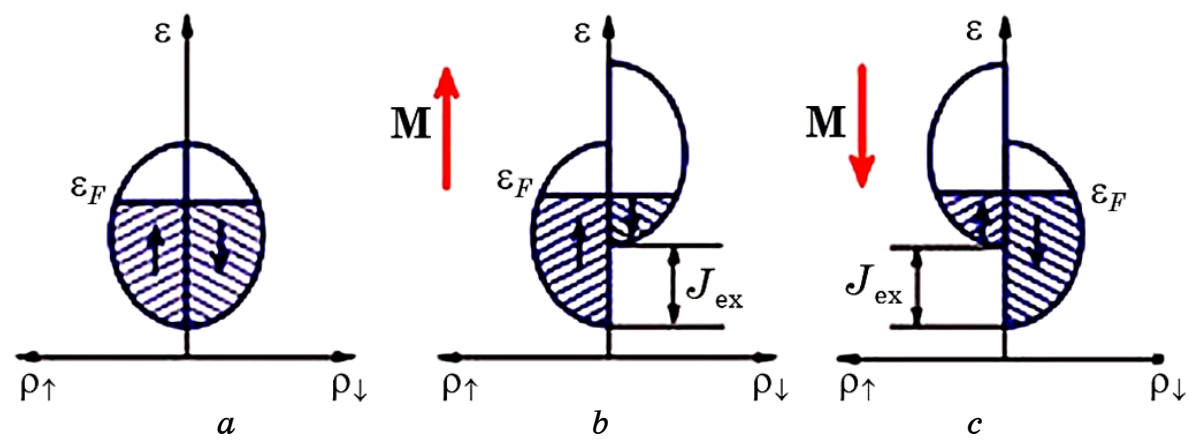

Fig. 1. Scheme of the spin splitting of the band spectrum caused by the exchange interaction $J_{\text {ex }}$, where $\rho \uparrow$ and $\rho \downarrow$ are the densities of electron states with different spin projections $(\uparrow \downarrow), \mathbf{M}$ is the magnetization vector: $a$-nonmagnetic metal, $b$-ferromagnetic metal, $c$-magnetic semimetals. 
In the mentioned case, the transfer electron charges into the controlled magnetic layer results in thermal losses and increase of a power consumption. This imposes restrictions on contact sizes, which have to provide the threshold density of the spin current subject to the condition that the electric current does not exceed the value of an electrical breakdown. These problems can be avoided by the utilizing the spin-orbit interaction for the spin polarization. In this case, the exchange interaction of the spin current with the localized spins is provided without passage of the charge current in the magnetic nanolayer [1-7].

The spin-orbit interaction in the two-dimensional systems with the broken structure inversion symmetry, known as the Rashba spin-orbit interaction [1,2,10-11], is realized in the interfaces of the magnetic nanostructures with two-dimensional electron properties and an interfacial potential drop. In a single-electron approximation, this interaction is described by the two-dimensional expression for a quasi-relativistic correction [2]. Taking into account the electric field $\mathbf{E}=E_{z} \mathbf{z}$ along growth direction $z$, the corresponding Rashba Hamiltonian can be represented as

$$
\mathbf{H}_{R}=\mathbf{B}_{R} \boldsymbol{\sigma}, \quad \mathbf{B}_{R}=\left(\frac{\alpha_{R}}{\hbar}[\mathbf{z} \times \mathbf{p}]\right),
$$

where $\alpha_{R} \propto E_{z}$ is the Rashba parameter, $\mathbf{B}_{R}$ is the effective Rashba magnetic field, which is dependent on the electron momentum p, and $\sigma$ is the vector of the Pauli spin matrices. Equation (1) describes the characteristic properties of the Rashba spin-orbit interaction, although in realistic systems, the broken inversion symmetry causes distorts of the free electron wave functions near to atomic nuclei and consequently, it changes a spin-orbit interaction [2]. Due to Eq. (1), the effective Rashba field $\mathbf{B}_{R}$ exerts the spin precession of the conduction electrons. In addition, one leads to the symmetric spin splitting of the single-electron dispersion along the conduction electron momentum that experimentally observed in interfaces of magnetic nanostructures (Fig. 2).

The spin-polarized electric current lies in the plane of the magnetic layer and one does not pass in the normal direction. Its interaction with the localized magnetization occurs through $s-d$ exchange interaction in the form of $\mathbf{H}_{s d}=J_{s d} \mathbf{S} \sigma$, where $J_{s d} \propto J_{c}$, where $J_{c}$ is the input electric current and $\mathbf{S}$ is the localized spin. Thereby, the change of the incoming electric current results in the corresponding change of the magnetization. The application of an electric field along the vector $\mathbf{z}$ results in changes of the Rashba parameter and magnetization dynamics, which is restricted by the magnetization switching.

The spin polarization of the electric current can be caused by the 


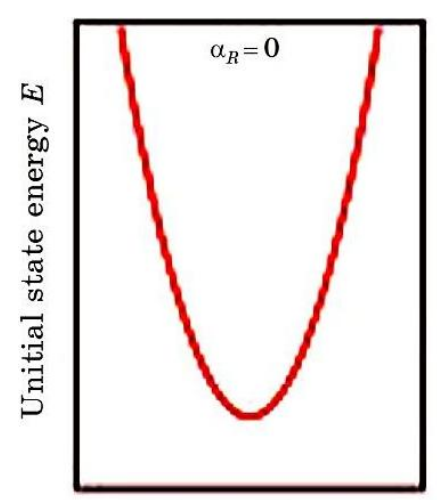

Wave vector $\mathbf{k}_{\|}$

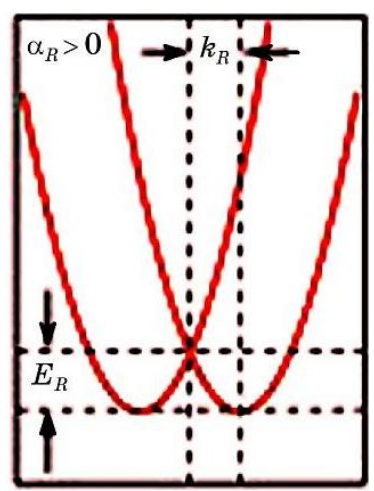

Wave vector $\mathbf{k}_{\|}$

Fig. 2. The dispersions of the two-dimensional electron gas previous to (on the left) and after (on the right) action of the Rashba spin-orbit interaction causing the spin-splitting along the planar momentum vector $\mathbf{k}_{\|} ; \alpha_{R}$ is the Rashba parameter, $k_{R}$ is the offset away from $\mathbf{k}_{\|}=\mathbf{0}$ of the spectral curves relative to the initial position.

spin Hall effect [7, 8], in which the passage of the electric current through heavy metal (for instance, $\mathrm{Pt}, \mathrm{Ta}$ ) with the strong spinorbit interaction exerts the spin dependent transverse deviation of the electric current and the transverse pure spin current. The spin orientation of the latter is perpendicular to the electric current and the interface normal (Fig. 3). The electric current lies in the plane of the adjacent heavy metal nanolayer and do not pass into the magnetic nanolayer. This avoids the mentioned constraints on contact sizes in the magnetic nanostructure and leads to reduction of the threshold current densities and the energy consumption.

The additional transverse momentum component $\mathbf{p}_{\sigma \perp}$ in the spin Hall effect is proportional to the derivative of the spin-orbit interaction $\mathbf{H}_{s o}=\beta[\nabla \mathbf{V} \times \mathbf{p}] \sigma$ with respect to the momentum $\mathbf{p}$, i.e. $\mathbf{p}_{\sigma \perp} \propto \beta$ (see [8]). The solution of the corresponding Schrödinger Hamiltonian with the spin-orbit interaction gives that the action of the latter on the conduction electrons is equivalent to the action of the effective spin-dependent Lorentz force $\mathbf{F}_{\sigma} \propto\left[\mathbf{v} \times \mathbf{B}_{\sigma}\right]$, where $\mathbf{v}$ is the electron velocity, and $\mathbf{B}_{\sigma}=\left[\nabla \times \mathbf{A}_{\sigma}\right]$ is the spin-dependent effective magnetic field. Here, $\mathbf{A}_{\sigma} \propto\left[\nabla \times \mathbf{E}_{\text {tot }}\right]$ is the effective magnetic potential with $\mathbf{E}_{\text {tot }}$ being the total electric field [8]. The effective field $\mathbf{B}_{\sigma}$ causes the transverse deviation of the electric current with generation of the transverse spin current. The charge current passes along the heavy-metal nanolayer and the generated spin current passes into the controlled magnetic layer where owing the exchange interaction through the torque changes the magnetization dynamics. This can lead to the magnetization precession or switching. 


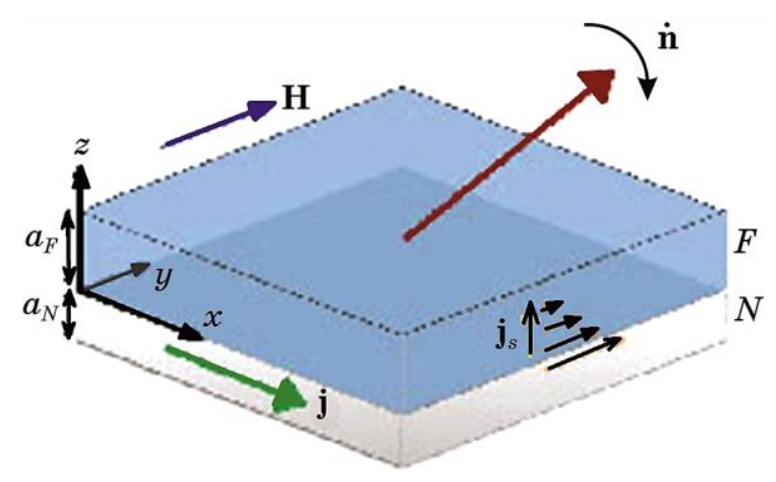

Fig. 3. The spin Hall effect of the spin-orbit conversion of the input electric current $\mathbf{j}$ in the transverse spin current $\mathbf{j}_{s}$ with the spin polarization denoted by arrows along the $y$ axis in the two-layered magnetic nanostructure. The latter is composed of the bottom layer made of heavy normal metal $(N)$ and the strong spin-orbit interaction and the upper ferromagnetic layer (FM). The spin current exerts on the dynamics of magnetization $\mathbf{n}$ of the magnetic layer; $\mathbf{H}$ is external magnetic field.

\section{THE SPIN-ORBIT-INDUCED TORQUE}

Current-driven magnetization by spin-orbit torques related to the nonequilibrium spin density of different origin subject to contributions of the interface spin-orbit Rashba effect and the bulk spin Hall effect in the layered magnetic nanostructure. Dependences of the corresponding Rashba $\left(\mathbf{T}_{R}\right)$ and spin Hall $\left(\mathbf{T}_{S H}\right)$ torques on the spin polarization and localized magnetization are similar, though the ratios of longitudinal and transverse components of each are different. The latter is related to features of their spin and charge density diffusion.

Two different mechanisms have been suggested and give rise to spin-orbit torque in bilayers consisting of a heavy-metal substrate and a thin ferromagnetic layer deposited on top of it. The first mechanism is attributed to the spin Hall effect [2] that generates a spin current from the substrate towards the ferromagnet. The second mechanism is due to the generation of a current-induced spin accumulation at the interface between the two materials, where magnetism, spin-orbit coupling, and broken inversion symmetry coexist.

The characteristic features of the spin-orbit torque in magnetic nanolayers can be described in the framework of the $s-d$ model Hamiltonian [10, 11]

$$
H=\left(\frac{\mathbf{p}^{2}}{2 m}+V(\mathbf{r})\right)-J_{s d}\left(\mathbf{s S}_{d}\right)+\frac{1}{m c^{2}}[\nabla \mathbf{V} \times \mathbf{p}] \mathbf{s},
$$


where the first bracket separated expression is the sum of kinetic and potential energy, the second term is the $s-d$-exchange interaction between itinerant electron spin $\mathbf{s}$ and the localized spin $\mathbf{S}_{d}$. Introduction of the spinor wave function $\Psi(\mathbf{r}, t)=\left[\Psi^{\uparrow}(\mathbf{r}, t), \Psi^{\downarrow}(\mathbf{r}, t)\right]$, spin current density magnetization $\mathbf{m}=\Psi^{*}(\mathbf{r}, t) \mathbf{s} \Psi(\mathbf{r}, t)$ and current density $\mathbf{J}_{s}=-(\hbar / m)\left[\Psi^{*}(\mathbf{r}, t) \mathbf{s} \nabla_{\mathbf{r}} \Psi^{*}(\mathbf{r}, t)\right]$ give equations, which after a quantum-mechanical averaging gives

$$
\begin{aligned}
& \frac{d \mathbf{m}}{d t}=\nabla J_{s}-\frac{J_{\mathrm{ex}}}{\hbar} \mathbf{M} \times \mathbf{m}+\frac{1}{m c^{2}}\langle[\nabla V \times \mathbf{p}] \times \mathbf{s}\rangle, \\
& \frac{d \mathbf{M}}{d t}=-\gamma \mathbf{M} \times \mathbf{H}+\alpha \mathbf{M} \times \frac{d \mathbf{M}}{d t}+\frac{J_{\mathrm{ex}}}{\hbar} \mathbf{M} \times \mathbf{m},
\end{aligned}
$$

where $\mathbf{M}$ is the unite vector of the localized magnetization, $\mathbf{H}$ is the effective field, $\gamma$ is the gyromagnetic ratio, $\alpha$ is the Gilbert damping. Here, the latter term in the first equation describes the spinorbit torque $\mathbf{T}$, which at a uniform magnetization $\left(\nabla J_{s}=0\right)$ takes the form as follows:

$$
\mathbf{T}=\frac{J_{\mathrm{ex}}}{\hbar} \mathbf{M} \times \mathbf{m}=\frac{1}{m c^{2}}\langle[\nabla V \times \mathbf{p}] \times \mathbf{s}\rangle .
$$

The spin-orbit torque can be represented in the general form:

$$
\mathbf{T}=\mathbf{T}_{\|}+\mathbf{T}_{\perp},
$$

where $\mathbf{T}_{\|}$is the field-like torque dominating in interfaces with the spin-orbit Rashba effect leading to magnetization switching. The damping-like torque $\mathbf{T}_{\perp}$ is small in interfaces but large in the bulk of magnetic layer where the spin Hall effect operates.

For ferromagnetic nanolayers,

$$
\mathbf{T}_{\|} \propto[\mathbf{y} \times \mathbf{M}], \quad \mathbf{T}_{\perp} \propto \mathbf{M} \times[\mathbf{y} \times \mathbf{M}]
$$

where $\mathbf{y}$ is the spin-polarization direction. Omitted coefficients in Eq. (6) are determined by features of spin and charge density diffusion, which have the form of the coupled system [11] in the twodimensional interfaces:

$$
\begin{gathered}
\frac{\partial n}{\partial t}=D \nabla^{2} n+B \nabla_{z} \mathbf{s}+\Gamma \nabla_{z} \mathbf{M} n+R \mathbf{M}(\mathbf{s} \mathbf{M})+R \mathbf{M}(\mathbf{s} \mathbf{M}) \\
\frac{\partial \mathbf{s}}{\partial t}=D \nabla^{2} \mathbf{s}-\frac{1}{\tau_{\|}} \mathbf{s}_{\|}-\frac{1}{\tau_{\perp}} \mathbf{s}_{\perp}+B \nabla_{z} \mathbf{s}-J_{s d} \mathbf{s} \times \mathbf{M}-\frac{1}{T_{s d}} \mathbf{M} \times(\mathbf{s} \times \mathbf{M})+B \nabla_{z} n+ \\
+2 C \nabla_{z} \times \mathbf{s}+2 R\left(\mathbf{M} \nabla_{z} n\right) \mathbf{M}+\Gamma\left[\mathbf{M} \times\left(\nabla_{z} \times \mathbf{s}\right)+\nabla_{z}(\mathbf{M} \times s)\right]
\end{gathered}
$$

Here, $n$ and $\mathbf{s}$ are the charge and spin densities, $\nabla_{z}=\mathbf{z} \times \nabla$. The spin 
density $\mathbf{S}_{\|}=s_{x} \mathbf{x}+s_{y} \mathbf{y}$ relaxes at a rate $1 / \tau_{s d}+1 / \tau_{s f}$ while $\mathbf{S}_{\perp}=s_{z} \mathbf{z}$ has a rate $1 / \tau_{\perp}=2 / \tau_{s d}+1 / \tau_{s f}$.

For a broad range of the relative strength between spin-orbit coupling and the exchange splitting, Eq. (7) and Eq. (8) describe spin dynamics in ferromagnetic layer. The $B$-term provides a source that generates spin density electrically. The $C$-term describes the coherent precession of the spin density around the effective Rashba field. The precession of the spin density (induced by the Rashba field) around the exchange field is described by the $\Gamma$-term. The $R$ term contributes to the magnetization renormalization.

\section{CONDITIONS OF A ROBUSTNESS OF MAGNETIC DYNAMICS}

In ferromagnet (FM)/normal metal (NM) heterostructures, spin pumping and spin-transfer torques are two reciprocal processes that occur concomitantly. Their interplay introduces a dynamic feedback effect interconnecting energy dissipation channels of both magnetization and current. The solution of the spin diffusion process in the presence of the spin Hall effect (SHE) in the normal metal shows that the dynamic feedback gives rise to a nonlinear magnetic damping that is crucial to sustain uniform steady-state oscillations of a spin Hall oscillator [14-17].

In FM/NM heterostructures, nonlocal effects arise because conduction electrons and magnetization reside in different materials and couple only at the interface. In this regime, spin pumping plays the role of spin electromotive force (SMF), which refers to the generation of spin current from a precession FM into the NM [16]. The pumped spin current is accompanied by a backflow of spin current $[15,16]$, which reacts on the FM through the spin transfer torque (STT). The combined effect of spin pumping and backflow-induced STT renormalizes the spin-mixing conductance at the interface [12, 14]. However, in the presence of the SHE, spin pumping and spin backflow are also connected by the combined effect of the SHE and its inverse process, which forms a feedback loop as illustrated in Fig. 4.

This additional feedback mechanism, proportional to $\theta_{S}^{2}$, where $\theta_{S}$ is the spin Hall angle, that is essential to the electron transport in FM/NM heterostructures. Consequently, this feedback effect is important to the magnetization dynamics. In a reciprocal sense, if we apply an ac current density to the NM, the SHE will drive the magnetization precession via the STT, which in turn can pump spin current back into the NM and renormalize the resistivity by means of the inverse SHE.

The feedback effect qualitatively modifies the dynamical behaviour of an FM/NM heterostructure. The feedback manifests as a 


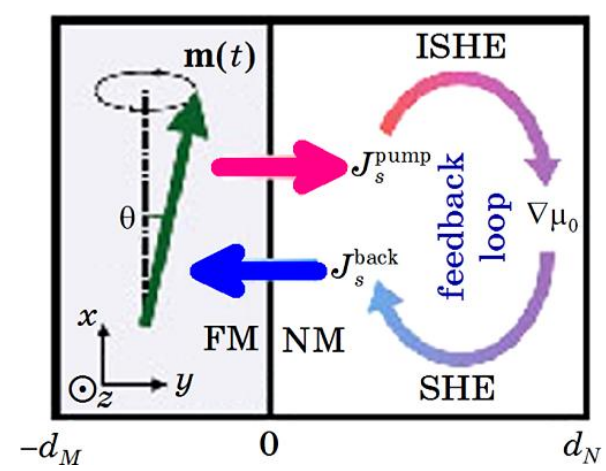

Fig. 4. In a FM/NM bilayer, spin pumping and spin backflow are connected by the SHE and it inverse process (ISHE).

novel nonlinear damping effect in the magnetization dynamics. It enables uniform auto-oscillations of a spin Hall oscillator and prevents magnetic switching. The feedback effect gives rise to a spin Hall magnetoimpedance in the electron transport, which reduces to the observed SMR in the dc limit.

Consider a FM/NM bilayer structure as shown in Fig. 4, where the layer thicknesses are $d_{M}$ and $d_{N}$, respectively. The coordinate system is chosen such that the magnetization direction at rest is along $x$ direction, and the interface normal is along $z$ direction. It is assumed that the FM is insulating (e.g., YIG), but the essential physics remains valid for a conducting FM since the feedback process takes place only on the NM side. Let $\mu_{0} / 2$ be the electrochemical potential and $\mu$ the vector of spin accumulation in the NM. The charge $\left(J_{i}^{C}\right)$ and the spin $\left(J_{i j}^{S}\right)$ current densities are

$$
J_{i}^{C}=-\frac{\sigma}{2 c}\left[\partial_{i} \mu_{0}-\theta_{S} \varepsilon_{i j k} \partial_{j} \mu_{k}\right] \text { and } J_{i j}^{S}=-\frac{\sigma}{2 c}\left[\partial_{i} \mu_{j}-\theta_{S} \varepsilon_{i j k} \partial_{k} \mu_{0}\right],
$$

respectively, with $i$ denoting the transport direction and $j$ denoting the direction of spin polarization. In given device geometry, only the spin current flowing along $z$-direction is relevant, thus we assume $\mu=\mu(z, t)$. Correspondingly, the spin current density reduces to a vector $J_{S}$; we scale it in the same unit as the charge current density $J_{C}$. The electron and spin dynamics in the NM are described by three equations

$$
\begin{gathered}
\frac{\partial \mu}{\partial t}=D \frac{\partial^{2} \mu}{\partial z^{2}}-\frac{1}{\tau_{s f}} \mu, \\
J_{C}=-\frac{\sigma}{2 e}\left[\nabla \mu_{0}+\theta_{S} z \times \frac{\partial \mu}{\partial z}\right],
\end{gathered}
$$




$$
J_{S}=-\frac{\sigma}{2 e}\left[\frac{\partial \mu}{\partial z}+\nabla \mu_{0}+\theta_{S} z \times \frac{\partial \mu_{0}}{\partial z}\right],
$$

where $D$ is the diffusion constant, $\tau_{s f}$ is the spin-flip relaxation time, $\sigma$ is the conductivity, $e$ is the electron charge, and $\theta_{S}$ is the spin Hall angle.

To solve the spin accumulation $\mu$, we assume that the charge current density $\mathbf{J}_{C}$ is fixed by external circuit and is uniform in space. Besides that, we have two boundary conditions: $\mathbf{J}_{S}\left(d_{N}\right) \equiv \mathbf{J}_{S 0}=\mathbf{0}$ and

$$
\mathbf{J}_{S 0}=\frac{G_{r}}{e}\left(\mathbf{M} \times\left(\mathbf{M} \times \mu_{S 0}\right)+\hbar \mathbf{M} \times \dot{\mathbf{M}}\right),
$$

where $\mu_{S 0}=\mu(0)$ and $G_{r}$ is the real part of the areal density of the spin-mixing conductance (the imaginary part is the real part of the areal density of the spin-mixing conductance (the imaginary part $G_{i}$ is neglected since $G_{r} \gg G_{i}$ ). On the right hand side of Eq. (12), the first term is the STT and the second term is the spin pumping. They are two fundamental ingredients bridging the electron (spin) transport in the NM with the magnetization dynamics of the FM. Due to the conservation of spin angular momentum, the spin current density $\mathbf{J}_{S 0}$ is absorbed by the FM, which is reflected by the Landau-Lifshitz-Gilbert (LLG) equation:

$$
\frac{d \mathbf{M}}{d t}=\gamma \mathbf{H}_{\mathrm{eff}} \times \mathbf{M}+\alpha_{0} \mathbf{M} \times \frac{d \mathbf{M}}{d t}+\frac{\hbar \gamma_{\mathrm{ex}}}{2 e M_{S} d_{M}} \mathbf{J}_{S 0},
$$

where $\gamma$ is the gyromagnetic ratio, $\hbar$ is the reduced Planck constant, $M_{S}$ is the saturation magnetization, $\alpha_{0}$ is the Gilbert damping constant, and $\mathbf{H}_{\text {eff }}$ is the effective magnetic field. For typical FMs, the magnetization dynamics is much slower than the spin relaxation rate in the $\mathrm{NM}$ so that $\omega r_{s f}<1$. In this limit, the spin accumulation $\mu(z, t)$ adapts to the instantaneous magnetization orientation and is kept quasi-equilibrium. As a result, the spin dynamics described by Eq. (9) reduces to a stationary spin diffusion process at any specified time. Retaining to second order in $\theta^{2}{ }_{S}$, Eq. (9) is solved as

$$
\mu(z)=\theta_{z} \frac{2 e \lambda}{\sigma} \mathbf{z} \times \mathbf{J}_{c} \frac{\operatorname{sh} \frac{2 z-d_{N}}{2 \lambda}}{\operatorname{ch} \frac{d_{N}}{2 \lambda}}+\frac{2 e \lambda}{\sigma}\left[\mathbf{J}_{S 0}+\theta_{S}^{2} \mathbf{z} \times\left(\mathbf{z} \times \mathbf{J}_{S 0}\right)\right] \frac{\operatorname{ch} \frac{z-d_{N}}{\lambda}}{\operatorname{sh} \frac{d_{N}}{\lambda}},(
$$

where $\lambda=\sqrt{D \tau_{s f}}$ is the spin diffusion length. Here, it is suppressed the $t$ variable in $\mu(z)$ since its time dependence simply originates from $\mathbf{J}_{C}$ and $\mathbf{J}_{S 0}$. Combining Eq. (9), Eq. (14), we can either eliminate the electron degrees of freedom $\left(\mathbf{J}_{C}\right.$ and $\left.\mathbf{J}_{S 0}\right)$ to derive an effective dynamics of the magnetization, or eliminate the time derivative 
of the magnetization (M) to get an effective magnetotransport of the electrons. These operations amount to invoking the dynamic feedback mechanism.

Assume that $\mathbf{J}_{C}$ is an applied dc charge current density. Our goal is to express the total spin current density $\mathbf{J}_{S 0}$ flowing into the $\mathbf{F M}$ in terms of the magnetization $\mathbf{M}(t)$, by which the LLG Eq. (13) will no longer involve any electron degrees of freedom, and the feedback effect is thus implemented mathematically. To this end, we combine Eq. (12) and Eq. (13), which gives two convoluted relations of $\mathbf{J}_{S 0}$ and $\mu_{\mathrm{S} 0}$. By means of iterations truncating at $\theta^{2}{ }_{S}$ order, we can solve $\mathbf{J}_{S 0}$ as a function of $\mathbf{J}_{C}, \mathbf{M}(t)$ and its time derivative. Then, we insert this $\mathbf{J}_{C}$ into Eq. (13), which yields the effective magnetization dynamics:

$$
\begin{gathered}
\frac{d \mathbf{M}}{d t}=\gamma \mathbf{H}_{\text {eff }} \times \mathbf{M}+\omega_{S} \mathbf{M} \times\left[\left(\mathbf{z} \times \mathbf{J}_{S 0}\right) \times \mathbf{M}\right]+ \\
+\left(\alpha_{0}+\alpha_{s p}\right) \mathbf{M} \times \frac{d \mathbf{M}}{d t}+\alpha_{r b}\left(\mathbf{M}_{S}^{2} \mathbf{M} \times \mathbf{z}\right),
\end{gathered}
$$

where $\mathbf{j}_{S}$ is the unite vector of $\mathbf{J}_{C}$, and

$$
\omega_{S}=\theta_{S} J_{C} \frac{\hbar \gamma}{e M_{S} d_{M}} \frac{\lambda G_{r} \operatorname{th} \frac{d_{s}}{2 \lambda}}{\sigma+2 \lambda G_{r} \operatorname{cth} \frac{d_{s}}{\lambda}}
$$

is the strength of the STT (driven by $\mathbf{J}_{C}$ ) scaled in the frequency dimension. The two damping coefficients are

$$
\begin{gathered}
a_{s p}=\frac{\hbar^{2} \gamma}{2 e M_{S} d_{M}} \frac{\sigma_{r} G_{r}}{\sigma+2 \lambda G_{r} \operatorname{ch} \frac{d_{N}}{\lambda}}, \\
a_{f b}=\theta_{S}^{2} \frac{\hbar^{2} \gamma}{e^{2} M_{S} d_{M}} \frac{\sigma_{r} \lambda G_{r}^{2} \operatorname{cth} \frac{d_{N}}{\lambda}}{\sigma+2 \lambda G_{r} \operatorname{cth} \frac{d_{N}}{\lambda}},
\end{gathered}
$$

Here, $a_{s p}$ describes the conventional enhanced damping from spin pumping with the spin backflow effects taken into account [16]; it is independent of the SHE. By contrast, the $a_{s p}$ term is completely new. It reflects the dynamic feedback realized by virtue of the combined effect of the SHE and its inverse process as schematically shown in Fig. 11. Equation (15) shows that this novel damping term is nonlinear in $\mathbf{M}_{\perp}$ : the component of $\mathbf{M}$ transverse to the effective field $H_{\text {eff }}$, whereas the Gilbert damping term is linear in $\mathbf{M}_{\perp}$.

The feedback-induced nonlinear damping effect can be understood in an intuitive way. If the magnetization precession is getting lar- 
ger, it will trigger a chain reaction: first, the pumped spin current $\mathbf{J}_{S 0}$ increases, then the spin diffusion becomes stronger (i.e., $\left|\partial_{z} \mu_{S}\right|$ gets larger). This will necessarily lead to a larger $\nabla \mu_{0}$ in the NM according to Eq. (10), as we have fixed the current density $\mathbf{J}_{S}$. Finally, the change of the emf will feed back into $\mathbf{J}_{S 0}$ according to Eq. (11), preventing its further increase. As a consequence, the growing magnetization precession is inhibited. The entire process realizes a negative feedback and respects Lenz's law.

\section{INTERCONNECTION OF SPIN CURRENTS AND MAGNETIC DYNAMICS IN AF BASED NANOSTRUCTURES}

The spin pumping and spin-transfer torque in AF based nanostructures represent the combined effect of their action in each of magnetic sublattices of the AF coupled by a strong exchange interaction. Magnetization dynamics of these coupled sublattices leads to an $\mathrm{AF}$ order (1) dynamics manifesting as precession and switching. Similarly, to the magnetization in the ferromagnetic case, the AF order precession generates the spin pumping current, which via the inverse spin Hall effect in adjacent nonmagnetic nanolayers can converts into the transverse charge current. Thereby, the influence of the AF dynamics on the charge current in AF nanostructures is realized [17]. The inverse impact of the charge current on the AF precession is realized via the spin Hall effect of the conversation of the charge current into the transverse spin current that owing to the exchange interaction exerts the spin transfer torque on the AF order precession.

Characteristic features of the AF dynamics and its interconnection with the spin currents in the form of the precession-induced spin pump and spin transfer torque is manifested in the AF twosublattice model with an easy axis is directed along the axis $z$ with magnetization unite vectors $\mathbf{m}_{1}$ and $\mathbf{m}_{2}$. These vectors are driven by the exchange interaction, the anisotropy, and a magnetic field in the $z$ direction. In units of frequency, they are represented by $\omega_{F}$, $\omega_{A}$, and $\omega_{H}=\gamma H_{0}$, respectively. The equations of motion in a free precession approximation are

$$
\dot{\mathbf{m}}_{1}=\mathbf{m}_{1} \times\left[\omega_{E} \mathbf{m}_{2}-\left(\omega_{E}+\omega_{H}\right) \mathbf{z}\right]+\mathbf{T}_{1}, \dot{\mathbf{m}}_{2}=\mathbf{m}_{2} \times\left[\omega_{E} \mathbf{m}_{1}-\left(\omega_{E}-\omega_{H}\right) \mathbf{z}\right]+\mathbf{T}_{2},
$$

where the effective field causing the magnetization precession in a magnetic sublattice contains the contribution from the exchange interaction with an adjacent magnetic sublattice. In line response, when $\mathbf{m}_{1(2)}=\mathbf{z}+\mathbf{m}_{1(2) \perp} e^{i \omega t}$ at $\left|\mathbf{m}_{\perp}\right|<<1$, the resonance frequencies are then 


$$
\omega=\omega_{H} \pm \omega_{R}=\omega_{H} \pm \sqrt{\omega_{A}\left(\omega_{A} \pm 2 \omega_{E}\right)},
$$

where the two corresponding eigenmodes are characterized by different chiralities. The left-handed (right-handed) mode, both $\mathbf{m}_{1}$ and $\mathbf{m}_{2}$ undergo a circular clockwise (counterclockwise) precession with $\pi$ phase difference. In the absence of magnetic field, viz. $\omega_{H}=0$, the two modes are degenerate.

Due to grasp, the essential feature of spin pumping by AF is to consider $\mathbf{m}_{1}$ and $\mathbf{m}_{2}$ as two independent $\mathbf{F}$ subsystems. Then, spin currents pumped from them will be proportional to $\mathbf{m}_{1} \times \dot{\mathbf{m}}_{1}$ and $\mathbf{m}_{2} \times \dot{\mathbf{m}}_{2}$, respectively. Since from $\mathbf{m}_{1} \approx-\mathbf{m}_{2}$ and $\dot{\mathbf{m}}_{1} \approx-\dot{\mathbf{m}}_{2}$, the total spin current is roughly proportional to $\mathbf{l} \times \mathbf{i}$, where $\mathbf{l}=\left(\mathbf{m}_{1}-\mathbf{m}_{2}\right) / \mathbf{2}$ is the staggered field. However, a more careful analysis reveals that the cone angles of $\mathbf{m}_{1}$ and $\mathbf{m}_{2}$ are different: in the left-handed (right-handed) mode, $\theta_{2} / \theta_{1}=\eta\left(\theta_{1} / \theta_{2}=\eta\right)$, where $\eta \approx\left(1+\sqrt{\omega_{A} / \omega_{E}}\right)^{2}$, so that a small magnetization $\mathbf{m}=\left(\mathbf{m}_{1}+\mathbf{m}_{2}\right) / 2$ will be induced in the AF dynamics state.

The spin currents in AF nanostructure determined by mixed scattering channels associated with different sublattices on the N/AF. Typical AF materials are insulators and incident electrons from the normal metal cannot penetrate far. Only a single atomic layer of AF directly connected to $\mathrm{N}$ suffices to describe the dominant contribution to interface scattering. Therefore, the essential physics is captured by modelling the N/AF interface as being semi-infinite in the transport direction and infinite in the transverse direction.

In the nearest-neighbour tight-binding model on a cubic lattice, in terms of the hopping energy in $\mathrm{N}$ and $\mathrm{AF} t$ and $t_{m}$, respectively, and the exchange coupling $J$ between the conduction electron spins and the magnetic moments, in the linear approximation in the small the small $\mathbf{m}$, the scattering matrix $S$ is

$$
S=S_{0}+S_{\omega} \tau_{1} \sigma_{0}+\Delta S\left[\tau_{3}(\mathbf{l} \cdot \sigma)+\tau_{0}(\mathbf{m} \cdot \sigma)\right],
$$

where $\tau_{1,2,3}$ are pseudospin Pauli matrices for sublattice degree of freedom, $\sigma$ are the vector of spin Pauli matrices, and $\tau_{0}$ and $\sigma_{0}$ are identity matrices. The last two terms of Eq. (21) with a common coefficient $\Delta S$ are spin dependent and represent the umklapp scatterings and normal ones, respectively. Pumping currents are related to the coefficients in Eq. (21) through the spin-mixing conductance,

$$
\begin{gathered}
G_{\text {mix }}=G_{r}+i G_{i}, \\
G_{r}=\left(e^{2} A / \hbar \pi^{2}\right) \iint|\Delta S|^{2} d k_{y} d k_{z}, \quad G_{i}=\left(e^{2} A / \hbar \pi^{2}\right) \iint \operatorname{Im}\left|S_{0}^{*} \Delta S\right| d k_{y} d k_{z},
\end{gathered}
$$


where $d k_{y}$ and $d k_{z}$ are the transverse momentums and $A$ the interface cross section. The conductance is determined by the scattering matrices with spin flip on the N/AF interface. By integrating over the Fermi surface, it can be shown that spin transfer on a compensated N/AF interface is similar in magnitude to the case of an uncompensated $\mathrm{N} / \mathrm{F}$ interface. This implies a similarity between the natures of spin pumping and spins transfer torque effects in $\mathrm{AF}$ and $\mathrm{F}$ nanosystems.

Although the AF resonance frequency reaches the $\mathrm{THz}$ region (1$10 \mathrm{meV}$ ), the motion of the staggered field remains adiabatic, as evidenced by comparing the resonance frequency with two characteristic energy scales: (i) the Fermi energy in $\mathrm{N}$ is a few eV; (ii) the exchange coupling between conduction electron spins and magnetic moments can be as large as $\mathrm{eV}$. As a result, the spin eigenstates and the scattering matrix Eq. (21) adiabatically adapt to the instantaneous configuration of AFs. Regarding the staggered field $\mathbf{l}$ and the magnetization $\mathbf{m}$ as two independent adiabatic parameters [19], we obtain the pumped spin current $\mathbf{I}_{S}$ with the scattering matrix $S$ in Eq. (21)

$$
\frac{e}{\hbar} \boldsymbol{I}_{s}=G_{r}(\mathbf{l} \times \dot{\mathbf{i}}+\mathbf{m} \times \dot{\mathbf{m}})-G_{i} \dot{\mathbf{m}} .
$$

Equation (22) can indeed be interpreted as arising from a coherent sum of two independent $\mathbf{F}$ spin pumping contributions by $\mathbf{m}_{1}$ and $\mathbf{m}_{2}$. However, the spin-mixing conductance $G_{r}$ and $G_{i}$ are different from those of $\mathrm{F}$ due to the mixing of scattering channels from different magnetic sublattices. Moreover, AF dynamics is much faster than $\mathrm{F}$ that corresponds to a stronger spin pumping.

By taking a time average of Eq. (22) over one period of oscillation, only the first two terms survive and contribute to the dc component of spin current $I_{s}^{\mathrm{dc}}$. Despite that $|\mathbf{m}|<<|\mathbf{l}|$, the contribution of $\mathbf{m} \times \mathbf{m}$ to $I_{s}^{\mathrm{dc}}$ can be comparable to that of $\mathbf{l} \times \mathbf{1}$. This is because $I_{s}^{\mathrm{dc}}$ is proportional to the cone angle $\theta^{2}$ of precession and the cone angle associated with the staggered field is much smaller than the one associated with the magnetization, $\theta_{l} \approx 0$ but $\theta_{m} \approx \pi / 2$.

From the sublattice degree of freedom involved in the AF dynamics, it follows a staggered spin pumping. A staggered spin current represents the imbalance between the spin current carried by the two sublattices. It has three components $\boldsymbol{I}_{s s}^{(i)}(i=1,2,3)$ associated with three pseudospin Pauli matrices. In a similar manner as spin pumping, we find that

$$
\frac{e}{\hbar} \mathbf{I}_{s s}^{(3)}=G_{r}(\mathbf{l} \times \dot{\mathbf{m}}+\mathbf{m} \times \dot{\mathbf{l}})-G_{i} \dot{\mathbf{i}}, \frac{e}{\hbar} \mathbf{I}_{s s}^{(2)}=-\operatorname{Re}\left[G_{\omega}\right] \dot{\mathbf{i}}, \frac{e}{\hbar} \mathbf{I}_{s s}^{(1)}=-\operatorname{Im}\left[G_{\omega}\right] \dot{\mathbf{m}},
$$


where

$$
G_{\omega}=\left(e^{2} A / \hbar \pi^{2}\right) \iint \operatorname{Im}\left|S_{\omega}^{*} \Delta S\right| d k_{y} d k_{z}
$$

results from intersublattice scattering that is unique to AFs. After the time average, $\mathbf{I}_{s s}^{(1)}$ and $\mathbf{I}_{s s}^{(2)}$ drop out, only $\mathbf{I}_{s s}^{(3)}$ survives. This time, the dc component $\boldsymbol{I}_{s s}^{\mathrm{dc}}$ is an even function of $\omega$ in the absence of static magnetic field. Elastic scattering in the normal metal will destroy any staggered spin accumulation, which decays on the time scale of $\hbar / t$. Therefore, the staggered spin current can only be well defined within a distance of the mean free path away from the interface.

The reciprocal effect of spin pumping is STT, which describes the back action that a spin current exerts on the AF. In linear response, an $\mathrm{AF}$ is driven by two thermodynamic forces $\mathbf{f}_{l}=-\delta F / \delta \mathbf{l}$ and $\mathbf{f}_{m}=-\delta F / \delta \mathbf{m}$ (energy dimension), where

$$
F=(\hbar / 2) \int d v\left[\frac{\omega_{0} \mathbf{m}^{2}}{a^{2}}+\omega_{l} \sum_{i=x, y, z} \frac{\left(\partial_{i} \mathbf{l}\right)^{2}}{a \omega_{H}}-\frac{\omega_{H} \mathbf{H m}}{(H a)^{3}}\right]
$$

is the free energy. Here, $\omega_{0}$ and $\omega_{n}$ are the homogeneous and inhomogeneous exchange frequencies, respectively. It can be easily shown that $\omega_{0}=\omega_{A}+2 \omega_{E}$. Enforced by $\mathbf{m l}=0$ and $(\mathbf{l})^{2} \approx 1$, the symmetry allowed dynamics are $\hbar \mathbf{i}=\left(a^{3} / v\right) \mathbf{f}_{m} \times \mathbf{l}$ and $\hbar \dot{\mathbf{m}}=\left(a^{3} / v\right) \mathbf{f}_{l} \times \mathbf{l}+$ $+\mathbf{f}_{m} \times \mathbf{m}$ [20], where $v$ is the system volume. Inserting them into Eq. (22) gives the response of the spin current to $\mathbf{f}_{m}$ and $\mathbf{f}_{l}$. Invoking the Onsager reciprocity relation, we derive the response of $\mathbf{l}$ and $\mathbf{m}$ to a given spin voltage $V_{s}$ in the normal metal, which are identified as two STT terms $\tau_{l}$ and $\tau_{m}$. To linear order in $\mathbf{m}$,

$$
\tau_{l}=\frac{a^{3}}{e v}\left[G_{r} \mathbf{l} \times\left(\mathbf{m} \times \mathbf{V}_{s}\right)-G_{i} \mathbf{l} \times \mathbf{V}_{s}\right], \quad \tau_{l}=\frac{a^{3}}{e v} G_{r} \mathbf{n} \times\left(\mathbf{m} \times \mathbf{V}_{s}\right),
$$

that treats STTs on the two sublattices as completely independent.

In solving the AF dynamics, it is instructive to eliminate $\mathbf{m}$ and derive a closed equation of motion in terms of 1 alone [21]. Truncating to linear order in $\mathbf{V}_{s}, \mathbf{m}$, and $\mathbf{l}$, we obtain the effective dynamics

$$
\mathbf{l} \times\left(\mathbf{i}+\alpha \omega_{0} \mathbf{i}+\omega_{R}^{2} \mathbf{l}_{\perp}\right)=\frac{\omega_{0} a^{3} G_{r}}{e v} \mathbf{l} \times\left(\mathbf{l} \times \mathbf{V}_{s}\right),
$$

where $\alpha$-the Gilbert damping constant, and $\mathbf{l}_{\perp}$ are perpendicular components of 1 with respect to the easy axis. Since the STT only acts on the interface and we consider a thin AF film, we have disregarded a possible nonuniform motion of 1. Solution of Eq. (25) for the spectrum in the case $\mathbf{V}_{s}$, which is collinear with the easy axis, 
has the form

$$
\frac{\omega}{\omega_{0}}=\frac{1}{2}\left[-i \alpha \pm \sqrt{-\alpha^{2}+\frac{4 \omega_{A}}{\omega_{0}}+\frac{4 i \alpha^{3} G_{r} V_{s}}{e v \omega_{0}}}\right] .
$$

For small $V_{s}$, $\omega$ has a negative imaginary part so that any perturbed motion will decay exponentially in time and the system is stable. However, a sufficiently large $V_{s}$ will flip the sign of $\operatorname{Im}[\omega]$, which makes the system unstable and marks the onset of uniform AF excitation. The condition $\operatorname{Im}[\omega]=0$ determines the threshold spin voltage $V_{s}^{t h}= \pm e v \alpha \omega_{l} /\left(a^{3} G_{r}\right)$, where $+(-)$ corresponds to the excitation of the right-handed (left-handed) mode. The chirality selection by the sign of the spin voltage is just consistent with the direction control of spin pumping by the microwave polarization. Since $G_{r}$ scales linearly with the interface area, $V_{s}^{\text {th }}$ scales linearly with the thickness of the AF layer.

In real experiments, a challenge arises from the large $\omega_{R}$, but we can still get reasonable $V_{s}^{\text {th }}$ by reducing the layer thickness. For $\mathrm{MnF}_{2}$ of few $\mathrm{nm}$ thick, the threshold spin voltage is estimated to be 10-100 $\mu \mathrm{V}$. The STT-driven AF dynamics suggests the feasibility of building a spin-torque nanooscillator using AFs, which generates a $\mathrm{THz}$ signal from a dc input without the need of static magnetic field.

\section{THE CURRENT-INDUCED DYNAMICS OF AFS IN SPIN HALL GEOMETRY}

In the framework of the current-induced dynamics of insulating antiferromagnets in a spin Hall geometry, sufficiently large in-plane currents perpendicular to the Néel AF order can trigger spontaneous oscillations at frequencies between the acoustic and the optical eigenmodes $[12,14,17]$. The direction of the driving current determines the chirality of the excitation. When the current exceeds a threshold, the combined effect of current-induced torques and spin pumping introduces a dynamic feedback that sustains steady-state oscillations with amplitudes controllable via the applied current. This permits to obtain the spin Hall (SH) nanooscillator with operating frequencies in $\mathrm{THz}$ range.

When an applied STT compensates the magnetic damping, the magnetization becomes unstable: it either switches to another direction or evolves into a steady-state oscillation. While the former improves writing operations in magnetic memory devices, the latter enables sustainable ac signal generation from dc inputs, giving rise to spin-torque oscillators (STOs) [22, 23]. In ferromagnets, currents 
or magnetic fields can tune the STO output frequency from the $\mathrm{MHz}$ to the $\mathrm{GHz}$ regime.

STOs can potentially be operated at much higher $\mathrm{THz}$ frequencies when antiferromagnets (AFs) replace ferromagnets. Two features make this possible: (1) the eigenfrequencies of typical AFs fall into the $\mathrm{THz}$ range; (2) an anti-damping STTs can trigger spontaneous excitations of an AF. While most AFs are insulators where STTs cannot be operated by passing through a current, the spin Hall effect (SHE) can produce STTs even when electrons do not flow through the magnet [16]. Therefore, integrating STOs with the SHE paves the way towards low-dissipation spin Hall nanooscillators (SHNOs).

However, to realize AF-based SHNOs, current-induced excitations should not grow indefinitely, but instead should evolve into steadystate oscillations and generate a substantial ac output. Although an AF under the action of an anti-damping STT does not suffer magnetic switching, its Néel AF vector experiences either no dynamics or a right-angle precession around the direction of the spin accumulation [5]. Since the oscillation amplitude is not continuously tuneable via the applied current, the device does not meet the requirements of an SHNO.

Steady-state oscillations are realizable in ferromagnetic STOs for the following reasons. In a spin-valve device, the angle dependence of the Gilbert damping and that of the anti-damping STT differ. As a result, when the driving current is above the threshold, there exists a unique angle where the two competing effects compensate; a steady-state oscillation is stabilized at that angle.

However, this feature is no longer active where the SHE creates the anti-damping STT. Therefore, one needs to introduce alternative mechanisms to prevent a spontaneous excitation from growing into magnetic switching. For instance, the bullet mode localized in space exhibits a strong noncollinearity that can sustain auto-oscillations. On the other hand, to enable uniform excitations, the dipolar interaction is required. However, the dipolar interaction is negligible in AFs where the magnetization is vanishingly small.

We will exploit an above-mentioned feedback mechanism to justify that a $\mathrm{THz}$ SHNO is realizable in an AF/heavy-metal heterostructure. The feedback effect originates from the combined effect of the SHE and its reverse process that connects the spin pumping with the spin backflow [24, 25], which is independent of the dipolar interaction. First, we determine the threshold of spontaneous excitations by solving the AF order dynamics in the linear response regime and relate the threshold to a current density by studying the SHE in the heavy metal. Then, we numerically explore the nonlinear Néel AF order dynamics beyond the threshold by con- 
sidering the feedback effect, and show that the feedback is indispensable to sustain uniform auto-oscillations. In the considerate case, $\mathrm{THz}$ SHNO generates a substantial ac voltage output with its amplitude continuously tuneable via the applied dc current.

For description of magnetic dynamics, we assume that the AF has the two-sublattice crystal structure with the sublattice magnetizations characterized by two unit vectors $\mathbf{M}_{A}$ and $\mathbf{M}_{B}$. We introduce the $A F$ vector $\mathbf{l}=\left(\mathbf{M}_{A}-\mathbf{M}_{B}\right) / 2$ and the small magnetization $\mathbf{M}=\left(\mathbf{M}_{A}+\right.$ $+\mathbf{M}_{B}$ )/2; they satisfy $\mathbf{M l}=\mathbf{0}$ and $\mathbf{M}_{A}^{2}+\mathbf{M}_{B}^{2}=1$. In the exchange limit, $M<<1$, thus $l \approx 1$ and $\mathbf{l i}=0$. The Cartesian coordinates are chosen such that the hard axis is along $\mathbf{z}$, and the in-plane easy-axis along $\mathbf{x}$. We scale everything in (positive) angular frequency, where the hard axis anisotropy is described by $\omega_{\perp}$, the easy in-plane anisotropy $\omega_{\|}$, and the Heisenberg exchange interaction $\omega_{E}$. In the macrospin description, the free energy

$$
F=-\hbar \omega_{E} \mathbf{l}^{2}-\hbar \omega_{\|} \frac{(\mathbf{x l})^{2}+(\mathbf{x} \mathbf{M})^{2}}{2}-\hbar \omega_{\perp} \mathbf{l}^{2}-\hbar \omega_{\|} \frac{(\mathbf{z l})^{2}+(\mathbf{z M})^{2}}{2},
$$

which defines two thermodynamic forces $\hbar \mathbf{f}_{l}=-\partial F / \partial \mathbf{l}, \hbar \mathbf{f}_{M}=-\partial F / \partial \mathbf{M}$. The coupled equations of motion are as follow:

$$
\begin{aligned}
& \dot{\mathbf{M}}=\mathbf{f}_{l} \times \mathbf{l}+\mathbf{f}_{M} \times \mathbf{M}+\alpha(\mathbf{M} \times \dot{\mathbf{M}}+\mathbf{l} \times \dot{\mathbf{l}})+\mathbf{T}_{M}, \\
& \left.\dot{\mathbf{l}}=\mathbf{f}_{M} \times \mathbf{l}+\mathbf{f}_{l} \times \mathbf{M}+\alpha(\mathbf{M} \times \dot{\mathbf{l}}+\mathbf{l} \times \dot{\mathbf{M}})+\mathbf{T}_{l}\right),
\end{aligned}
$$

where $\alpha$ is the Gilbert damping constant, $\mathbf{T}_{M}$ and $\mathbf{T}_{l}$ are the STTs given by [25]

$$
\mathbf{T}_{M}=\mathbf{l} \times\left(\boldsymbol{\omega}_{S} \times \mathbf{l}\right)+\mathbf{M} \times\left(\boldsymbol{\omega}_{S} \times \mathbf{M}\right), \mathbf{T}_{l}=\mathbf{l} \times\left(\boldsymbol{\omega}_{S} \times \mathbf{M}\right)+\mathbf{M} \times\left(\omega_{S} \times \mathbf{l}\right) .
$$

Here, $\omega_{S}$ is the vector of spin accumulation; its magnitude (in frequency unit) represents the STT strength. To derive the currentinduced excitations, we decompose the $\mathrm{AF}$ vector as $\mathbf{l}=\mathbf{l}_{\perp} e^{i \omega t}$, assuming $\left|\mathbf{l}_{\perp}\right|<<1$. Restricting to linear order in $\mathbf{l}_{\perp}$, we eliminate $\mathbf{M}$ in Eq. (26), and obtain the eigenfrequencies as

$$
\frac{\omega_{ \pm}}{\omega_{E}}=i \alpha+\left[\frac{\omega_{\perp}+2 \omega_{\|} \pm \sqrt{\omega_{ \pm}^{2}-4 \omega_{S}^{2}}}{\omega_{E}}-\alpha^{2}\right]^{1 / 2},
$$

where the $+(-)$ sign corresponds to the optical (acoustic) mode. Due to the latter equation, the dependences of the real and imaginary parts $\operatorname{Re}\left[\omega_{ \pm}\right]$and $\operatorname{Im}\left[\omega_{ \pm}\right]$are characterized by degenerate lower of which $\operatorname{Im}\left[\omega_{ \pm}\right]$remain degenerate and unaffected. However, at $\omega_{S}>\omega_{\perp} / 2$, $\operatorname{Im}\left[\omega_{ \pm}\right]$reduces (grows) rapidly, indicating that the damping is diminished (enhanced) by the STT. At the threshold [25], 


$$
\omega_{S}^{t h}=\sqrt{\frac{\omega_{\perp}^{2}}{4}+\alpha^{2}\left(2 \omega_{\perp}+\omega_{\perp}\right) \omega_{E}} .
$$

$\operatorname{Im}\left[\omega_{+}\right]$vanishes, which marks the onset of spontaneous excitation of the optical mode and the breakdown of the linear response approximation. The uniaxial symmetry enforces that $\operatorname{Im}\left[\omega_{+}\right]$also vanishes for $\omega_{S}^{t h}$ so that the auto-oscillation can be triggered by a reversed current as well.

In the absence of the hard-axis anisotropy $\left(\omega_{\perp}=0\right)$, the threshold Eq. (27) is linear in $\alpha$, so the anti-damping effect occurs when the STT is turned on. However, in the general case when $\omega_{\perp}>0$, the anti-damping effect appears only when $\omega_{S}>\omega_{\perp} / 2$. Vectors $\mathbf{M}_{A}$ and $\mathbf{M}_{B}$ always exhibit opposite chiralities, i.e., they rotates counterclockwise (clock-wise). However, at the degenerate point $\omega_{S}>\omega_{\perp} / 2$, the chirality of $\mathbf{M}_{A}\left(\mathbf{M}_{B}\right)$ in the optical (acoustic) mode reverses. Consequently, when $\omega_{S}>\omega_{\perp} / 2$, both $\mathbf{M}_{A}$ and $\mathbf{M}_{B}$, hence the Néel vector $\mathbf{1}$, all acquire the same chirality. At the threshold $\omega_{S}^{t h}$, the excited optical mode is right-handed. If $\omega_{S}$ changes sign, the optical mode is still excited, but its chirality becomes left-handed. These suggest that the direction of the current determines the chirality of the excitation.

Let us consider the critical-current two-layered nanostructure insulating AF/heavy normal metal (HM) with strong spin-orbit coupling (Fig. 5).

A current density $\mathbf{J}_{c}$ is applied along the $y$-direction; it is perpendicular to the Néel vector of the AF. The SHE in the HM generates anti-damping STTs to drive the Néel vector dynamics, which in turn pumps spin current back into the HM. The pumped spin current converts into a charge voltage due to the inverse SHE. By solving

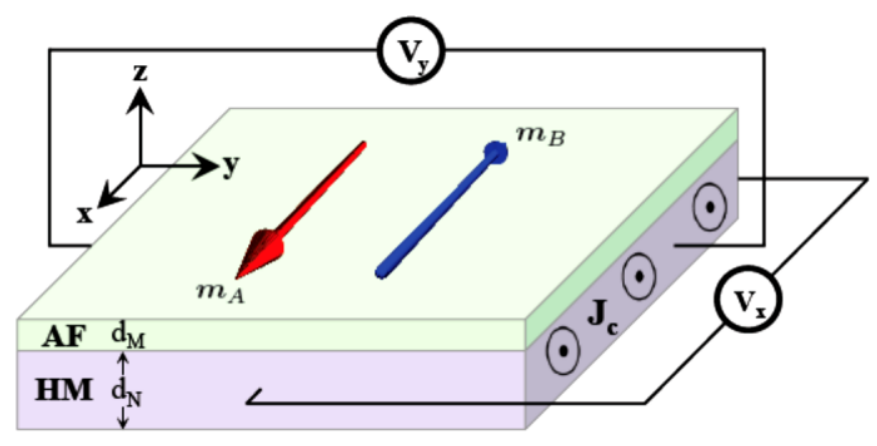

Fig. 5. An insulating AF/HM heterostructure. The applied dc current density $\mathbf{J}_{c}$ drives the AF via the SHE. The dynamics of the AF pumps spin current back into $\mathrm{N}$, and converts into electric field via the inverse SHE, which is monitored by two voltmeters. 
the spin diffusion equation in the presence of the SHE under boundary conditions involving both spin-pumping and STT, we relate the threshold STT Eq. (27) to a critical current density

$$
J_{c}^{t h}=\omega_{c}^{t h} \frac{d_{M}\left(h \sigma+2 \lambda e^{2} g_{r} \operatorname{cth} \frac{d_{N}}{\lambda}\right)}{2 \theta_{S} \alpha^{3} \lambda e g_{r} \operatorname{th} \frac{d_{N}}{\lambda}},
$$

where $\theta_{S}$ is the spin Hall angle, $g_{r}$ is the areal density of the transverse mixing conductance [25]. From Eq. (28), it is follows that the critical current density $J_{c}^{t h}$ can be lowered by reducing (increasing) the thickness of the $\mathrm{AF} d_{M}^{c}\left(\mathrm{HM} d_{M}\right)$.

The sustained steady-state oscillation of the Néel vector in the mentioned nanostructure can be realized via the dynamic feedback effect. The pumped spin current from a processing AF vector into the HM experiences a backflow [25]. In HMs, however, the spin pumping and the spin backflow are also connected via the combined effect of the SHE and its inverse process, which feeds the Néel vector dynamics back into itself. In ferromagnets, such a feedback mechanism manifests as a nonlinear damping effect in the magnetization dynamics. Similar feedback-induced damping effect can occurs for AFs. In this case, the pumped spin current into the HM converts into an electric field $\mathbf{E}$ due to the inverse SHE.

According to Ohm's law, $\mathbf{J}_{c}=\sigma \mathbf{E}-\theta_{S}[\sigma /(2 e)] \mathbf{z} \times \partial_{z} \mu_{S}$, where $\mu_{S}$ is the spin accumulation in the HM. At the fixed current density $\mathbf{J}_{c}$ through external circuits, a change of the electric field $\mathbf{E}$ necessarily leads to a change of the spin accumulation $\mu_{S}$. Subsequently, the change of $\mu_{S}$ diffuses and generates an additional spin current, which will finally deliver the influence of spin pumping back into the Néel vector through STTs. By closing such a feedback loop, we obtain a feedback torque that should be added to Eq. (27) as

$$
\mathbf{T}_{F B}=\alpha_{N L}\left(l_{z}^{2} \mathbf{l} \times \mathbf{i}-i_{z}(\mathbf{z} \times \mathbf{l})\right)
$$

where the feedback coefficient is

$$
a_{N L}=\theta_{s}^{2} \frac{\alpha^{3}}{d_{M}} \frac{2 \hbar \lambda e^{2} g_{r}^{2} \operatorname{cth} \frac{d_{N}}{\lambda}}{\left(\hbar \sigma+2 \lambda e^{2} g_{r} \operatorname{cth} \frac{d_{N}}{\lambda}\right)} .
$$

While the feedback effect seems to be a higher-order effect as $a_{N L}$ is proportional to $\theta_{S}^{2}$, it can be significantly enhanced by searching for materials with large $\theta_{S}$. The feedback-induced nonlinear damping is a critical ingredient because it dramatically modifies the dynamical behaviour of an SHNO using AF. 


\section{REFERENCES}

1. L. Zutic, J. Fabian, and S. Sarma, Rev. Mod. Phys., 76, No. 3: 323 (2004).

2. A. Manchon, H. C. Koo, J. Nitta, S. M. Frolov, and R. A. Duine, Nature Mater., 36, No. 7: 871 (2015).

3. J. Sinova, S. Valenzuela, J. Wunderlich, C. H. Back, and T. Jungwirth, Rev. Mod. Phys., 87, No. 6: 1213 (2015).

4. L. Liu, C.-F. Pai, Y. Li, H. W. Tseng, D. C. Ralph, and R. A. Buhrman, Science, 336: 555 (2012).

5. R. H. Liu, W. L. Lim, and S. Urazhdin, Phys. Rev. Lett., 110: 147601 (2013).

6. N. V. Volkov, Physics-Uspekhi, 55, No. 6: 263 (2012).

7. J. E. Hirsch, Phys. Rev. Lett., 83: 1834 (1999).

8. E. M. Chudnovsky, Phys. Rev. Lett., 99: 206601 (2007).

9. I. M. Miron, G. Gaudin, S. Auffer, B. Rodmacq, A. Schuhl, S. Pizzini, J. Vogel, and P. Gambardalla, Nature Matter., 9: 230 (2010).

10. A. Manchon and S. Zhang, Phys. Rev. B, 79, No. 10: 094422-1 (2009).

11. X. Wang, and A. Manchon, Phys. Rev. Lett., 108: 117201 (2012).

12. R. Cheng, J.-G. Zhu, and D. Xiao, arXiv: 01618v1 (2016).

13. H. V. Gomonay and V. M. Loktev, Phys. Rev. B, 81, No. 5: 144427 (2010).

14. R. Cheng and D. Xiao, arXiv: 1509.09229v2 (2016).

15. Ya. Tserkovnyak and S. A. Bender, Phys. Rev. B, 90, No. 7: 014428 (2014).

16. Ya. Tserkovnyak, A. Brataas, and E. W. Bauer, Phys. Rev. B, 66, No. 11: 224403 (2002).

17. R. Cheng, J. Xiao, Q. Niu, and A. Brataas, Phys. Rev. Lett., 113: 057601 (2014).

18. M. Hagiwara, K. Katsumata, I. Yamada, and H. Suzuki, J. Phys.: Condens. Matter, 8: 7349 (1996).

19. P. W. Brouwer, Phys. Rev. B, 58: R10135 (1998).

20. O. A. Tretiakov and A. Brataas, Phys. Rev. Lett., 110: 127208 (2013).

21. R. Cheng and Q. Niu, Phys. Rev. B, 86, No. 9: 245118 (2012).

22. K.-W. Kim, J.-H. Moon., K.-J. Lee, and H.-W. Lee, Phys. Rev. Lett., 108: 217202 (2012).

23. C. H. Wong and Ya. Tserkovnyak, Phys. Rev. B, 80, No. 12: 184411 (2009).

24. L. Berger, Phys Rev. B, 54, No. 15: 9353 (1996).

25. A. Brataas, Ya. Tserkovnyak, and E. W. Bauer, Phys. Rev. B, 66, No. 14: 060404 (2002). 